\title{
Thoracolumbar pseudarthrosis in ankylosing spondylitis
}

\begin{abstract}
Study design: Retrospective study of a series of 36 consecutive patients with thoracolumbar pseudarthrosis in ankylosing spondylitis (AS).

Objective: To analyze the clinical and radiological characteristics, especially the computed tomography (CT) with multiplanar reformations, of thoracolumbar pseudarthrosis in AS. A new classification of thoracolumbar pseudarthrosis in AS was put forward.
\end{abstract}

Summary of background data: Spinal pseudarthrosis (SP) is a well recognized complication in advanced AS. Its features are similar to infective spondylitis, so this may often result in misdiagnosis. The clinical and radiological characteristics (including plain film, CT with reconstruction and MRI) in a relative large series have not been reported because of the rarity of this condition.

Methods: During the period between Jan 2003 to Jan 2016, 36 patients with AS complicated thoracolumbar pseudarthrosis were treated in our department. Clinically, history of trauma and characteristics of symptoms were obtained by patient interviews. Radiologic assessment including plain film, CT with reconstruction and MRI were obtained in all patients. Their clinical and radiological characteristics were analyzed. A classification based on the $\mathrm{CT}$ with reconstruction was put forward.

Results: There were 31 males and 5 females, with an average age of 50.1 years (range 33 to 77 years). The diagnosis in all cases was further confirmed when the patients received surgery because of failure of conservative treatment. All of them complained of recurrent back pain and 12 complicated neurological deficits. Most lesions were near the thoracolumbar junction. There were two type of pseudarthrosis, trans-disc type and trans-vertebral type, based on the sclerotic bony gap of the pseudarthrosis. Twenty-eight of the 36 pseudarthrosis were trans-disc type and the other 8 were trans-vertebral type. Precedent traumatic events were found in all 8 cases with transvertebral type. Eighteen of 28 trans-disc type were found to have the characteristic of extensive discovertebral destruction on radiograph, but the other 18 cases (including 10 cases with trans-disc type and all 8 cases with trans-vertebral type) needed further radiological examinations for the diagnosis. CT with multiplanar reformation clearly characterized the pseudarthrosis, which showed continuous sclerotic bony gap through the three columns of thoracolumbar junction in all cases. MRI is superior in depicting spinal cord compression. The pathology further confirmed the diagnosis of SP in AS.

Conclusion: Two types of thoracolumbar pseudarthrosis, the trans-disc type and transvertebral type, can been identified in AS. More than half of the cases with trans-disc pseudarthrosis have the manifestation of extensive discovertebral destructive lesions on plain film. The continuous sclerotic gap across all columns of the spine showed on reformatted CT images is the characteristic of SP in AS. MRI is valuable in evaluating the spinal cord and the nerve roots in the cases with neurological deficits.

Keywords: thoracolumbar pseudarthrosis, ankylosing spondylitis, Andersson lesions, multiplanar reformatted computed tomography
Volume 10 Issue 6 - 2018

Ting Wang,' Bin Liu, ${ }^{2}$ Gang Jiang, ${ }^{3}$ Xiangyun Liu,' Yuanliang Sun,' Jianqiang Kou,' Xiujun Zheng'

'Department of Spinal Surgery, Affiliated Hospital of Qingdao University, China

${ }^{2}$ Department of Rheumatology, Affiliated Hospital of Qingdao University, China

${ }^{3}$ Department of Radiology, Affiliated Hospital of Qingdao University, China

Correspondence: Ting Wang, Department of Spinal Surgery, Affiliated Hospital of Qingdao University, Jiangsu Road 16, Qingdao, 266003, China, Tel 0086-0532-829II298, Fax 00860532-82911999, Email wangteng20@hotmail.com

Received: August 30, 2017| Published: November 28, 2018

\section{Introduction}

Spinal pseudarthrosis (SP) is a well recognized complication in advanced ankylosing spondylitis (AS). ${ }^{1}$ Such lesion may present with persistent back pain, which is exacerbated by exercise, and sometimes complicated with spinal deformities and neurological deficits. ${ }^{2}$ On plain film, it usually shows as extensive discovertebral destruction and occurs through the intervertebral disc spaces at the thoracolumar junction. In rare situation, it can be observed through the vertebral body. ${ }^{3}$ On magnetic resonance image (MRI), it is similar to infective spondylitis, so this may often result in misdiagnosis. ${ }^{46}$ There have been several reports describing the clinical and radiological findings of this condition. ${ }^{1-3,7,8}$ However, no reports have analyzed the clinical characteristics combined with a complete set of radiological examination (including plain film, CT with reconstruction and MRI) in a relative large series because of the rarity of this condition. In this report, we respectively reviewed the clinical and radiological features of thoracolumbar pseudarthrosis in AS in a consecutive cohort of patients. A new classification of thoracolumar pseudarthrosis in AS was put forward. 


\section{Methods}

Following approval by the institutional review board, 36 patients with AS complicated thoracolumbar pseudarthrosis who were treated in our department between Jan 2003 to Jan 2016 were respectively reviewed. Patients with acute traumatic fractures were excluded. All the cases received operation because of failure of conservative treatment. Clinically, history of trauma, characteristics of back pain and neurological deficits were obtained by patient interviews. Radiologic assessment including plain film, CT and MRI were obtained. Multiplanar CT reformations were especially used to assess the pseudarthrosis in all cases. Pathology of the pseudarthrosis was obtained in the 36 patients who received surgery. The diagnosis of all patients and radiologic assessment were made by a senior spine surgeon and a senior radiologist. A consensus on the standardized method of assessment was made prior to data collection.

\section{Results}

\section{Clinical findings}

There were 31 males and 5 females. The mean age was 50.1 years (range, 33-77 years). All patients had clearly established AS for more than 10 years by accepted clinical and radiological criteria. All patients complained of recurrent back pain after the disease had been quiescent for a period. The nature of the pain was mechanical, which were aggravated when standing or walking but relieved by lying down. Traumatic events that preceded development of symptoms were found in 8 cases. All the injuries were minor and had occurred at a mean of 13 months (3-26) previously. Of the 36 patients, 12 (33.3\%) had neurological deficits as Frankel C. All the 12 cases were trans-disc type pseudarthrosis. The details of the patients are shown in Table 1 and Table 2.
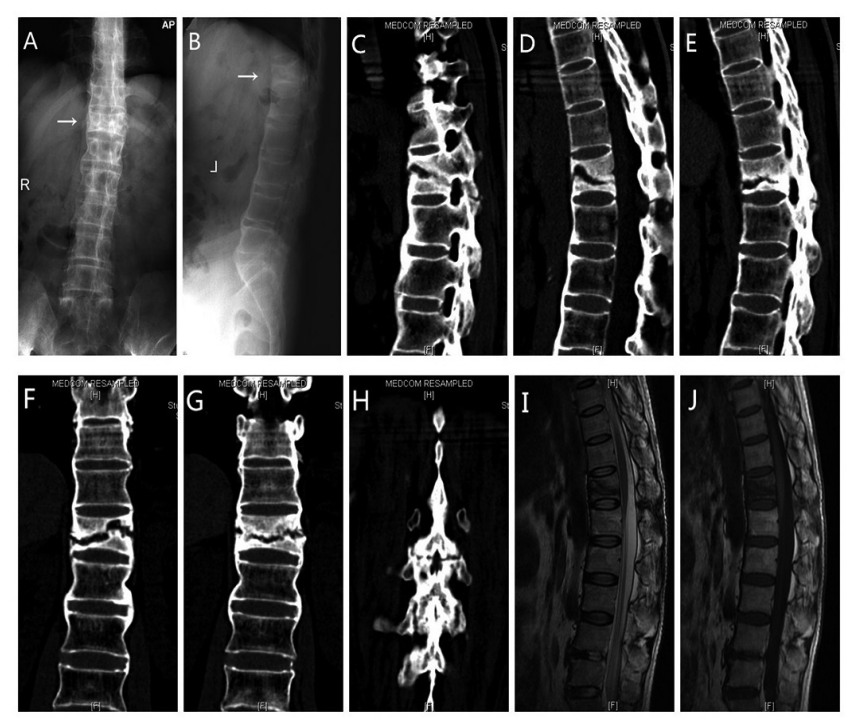

Figure I A, B:AP and lateral radiographs in a patient showing a trans-vertebral pseudarthrosis at TI2. There was no extensive discovertebral destruction on the plain film. The sclerotic gap of the pseudarthrosis cannot be shown on the radiograph. C, D, E: Sequential sagittal CT reconstructions showing the sclerotic gaps across three columns of the spine. It is a trans-vertebral pseudarthrosis at TI2. F, G, H: Sequential coronal CT reconstructions clearly showing the sclerotic gaps at TI2. I, J: Sagittal TI-weighted and T2-weighted images.
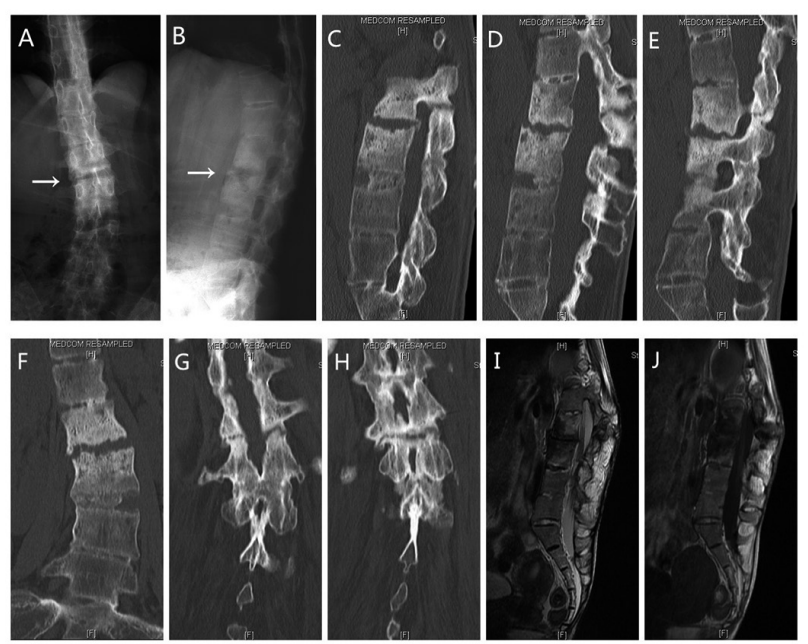

Figure $2 \mathrm{~A}, \mathrm{~B}: \mathrm{AP}$ and lateral radiographs showing a trans-disc pseudarthrosis at L2-L3. C, D, E: Sequential sagittal CT reconstructions showing the sclerotic gap across the three columns of the lumbar. F, G, H: Coronal CT reconstructions showing the pseudarthrosis across the spine. I, J: Sagittal TIweighted and T2-weighted images showing the pseudarthrosis.

\section{Radiologic findings}

All of the radiographs displayed typical changes in establishing AS, including fusion of the sacroiliac joints, and continuous syndesmophyte formation through the thoracic and lumbar spine. Most lesions were near the thoracolumbar junction with the highest at T8-9 and the lowest at L4-5. According the sclerotic bony gap, two types of pseudarthrosis can be classified, the trans-disc type and trans-vertebral type. Twenty-eight $(77.8 \%)$ lesions were trans-disc type and the other $8(22.2 \%)$ lesions were trans-vertebral type (Figure 1 \& Figure 2). All the 8 cases with trans-vertebral lesion had a history of trauma. Eighteen of the 28 cases with trans-disc lesions were found to have the characteristic of extensive discovertebral destruction (Andersson Lession) on plain film. The radiographic features of the extensive discovertebral destruction included the irregular disappearance of the vertebral end plate on both the upper and the lower border of the disc. The borders between discs were ragged and hazy instead of smooth and sharp. The diagnosis of pseudarthrosis could be made in these 18 cases with extensive discovertebral destruction on radiograph. In the other 18 , including 10 trans-disc type and all 8 trans-vertebral type, the lesions could be located on radiograph, but the diagnosis of pseudarthrosis needed further radiological examination.

CT with multiplanar reformation clearly characterized the pseudarthrosis, which showed continuous sclerotic bony gap through the three columns of thoracolumbar junction in all cases. Sequences of sagittal and coronal CT reformatted images showed the sclerotic lines were from anterior column (trans-disc or trans-vertebral), across the foraminae, to posterior elements. At the anterior column, vacuum phenomenons were detected in the disc or vertebral body in 16 cases $(44.4 \%)$, including 13 cases $(46.4 \%)$ in trans-disc type and 3 cases $(37.5 \%)$ in trans-vertebral type. Irregular osteolysis of the end plates and adjacent vertebral bodies was showed in the 18 cases with extensive discovertebral destructive lesions. At the middle column, all the sclerotic lines were through the foraminae except one trans-vertebral case through the pedicle. At the posterior column, either a sclerotic lines through the ankylosed posterior elements or 
unfused facet joints were identified. It was impossible to show the sclerotic line of the posterior elements on plain film because of the superimposition of heavily ossified spinal ligaments. The sagittal and coronal reformatted images were more useful than the axial scans in delineating the above changes. The encroachment of the spinal canal at the pseudarthrosis was assessed on the axial CT scan with reformatted images. They delineated the causes of the spinal stenosis mainly from hypertrophy of the posterior elements.

On MRI, both the trans-disc and trans-vertebral pseudarthrosis were identified. On T1-weighted image, all patients had irregular low signal band extended from anterior column into the posterior elements. It had two patterns: low signal on T1- and high signal on T2-weighted images (11 cases, including 8 cases of trans-disc type and 3 cases of trans-vertebral type), and low signal on both T1- and T2-weighted images ( 25 cases, including 20 cases of trans-disc type and 5 cases of trans-vertebral type). MRI was superior to CT in depicting spinal cord compression. In the 12 cases with neurological deficits, MRI showed the spinal cord sustained compression at the pseudarthrotic segment. It mainly resulted from hypertrophy of posterior pseudarthrosis, facet joints or ligamentum flavum (Figure 3 ). The segments of pseudarthrosis in the 12 cases with neurological deficits were all above L1 where spinal cord located.

Table I Clinical Details and Radiological Findings of Trans-disc Pseudarthrosis
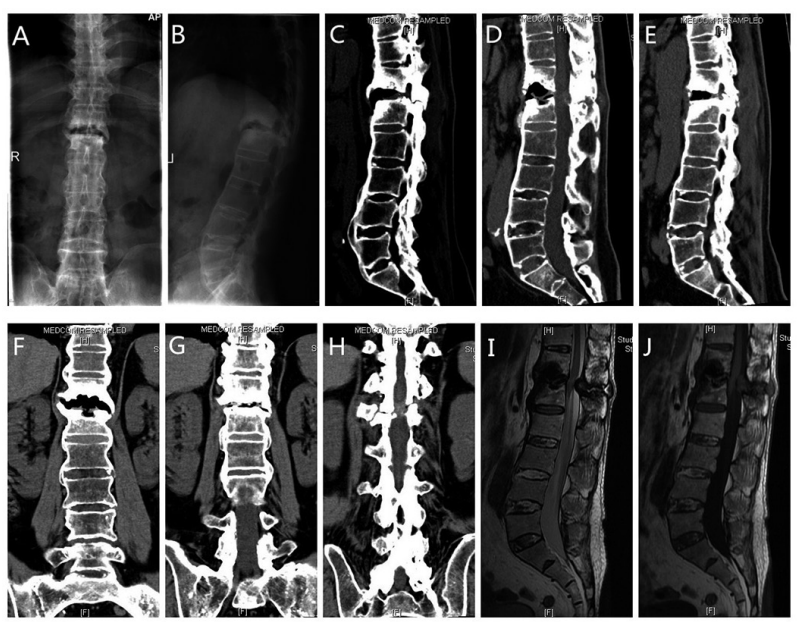

Figure $3 \mathrm{~A}, \mathrm{~B}$ : AP and lateral radiographs showing TI2-LI pseudarthrosis in a patient. There is extensive discovertebral destruction (Andersson lesion) at the intervertebral space with surrounding sclerosis. C, D, E: Sequential sagittal CT reconstructions showing the sclerotic gap from TI2-LI disc, across the foraminae, to the posterior element. F, G, H: Sequential coronal $\mathrm{CT}$ reconstructions showing the pseudarthrosis across three columns of the spine. I, J: Sagittal TI-weighted and T2-weighted images. The spinal cord is compressed mainly by hypertrophy of pseudarthrosis from the posterior element.

\begin{tabular}{|c|c|c|c|c|c|c|c|c|c|}
\hline Pts & $\begin{array}{l}\text { Age } \\
(y r)\end{array}$ & Sex & $\begin{array}{l}\text { Trau- } \\
\text { Histr }\end{array}$ & $\begin{array}{l}\text { Neuro- } \\
\text { deficits }\end{array}$ & $\begin{array}{l}\text { Pseud- } \\
\text { location }\end{array}$ & $\begin{array}{l}\text { Extensive } \\
\text { destructive } \\
\text { lesion }\end{array}$ & $\begin{array}{l}\text { Vacuum } \\
\text { phenomenon }\end{array}$ & TI-image & $\begin{array}{l}\text { T2- } \\
\text { image }\end{array}$ \\
\hline I & 68 & $M$ & No & No & L4-L5 & No & No & Low & Low \\
\hline 2 & 48 & $M$ & No & No & L4-L5 & Yes & No & Low & High \\
\hline 3 & 43 & $\mathrm{~F}$ & No & No & TI2-LI & Yes & No & Low & High \\
\hline 4 & 49 & $M$ & No & Yes & TIO-TII & Yes & No & Low & Low \\
\hline 5 & 53 & $M$ & No & Yes & TI2-LI & Yes & Yes & Low & Low \\
\hline 6 & 39 & $\mathrm{~F}$ & No & No & L2-L3 & Yes & No & Low & Low \\
\hline 7 & 51 & $M$ & No & Yes & TIO-TII & Yes & Yes & Low & Low \\
\hline 8 & 33 & $M$ & No & No & TI2-LI & Yes & No & Low & Low \\
\hline 9 & 48 & $M$ & No & Yes & T9-TI0 & Yes & No & Low & Low \\
\hline 10 & $4 I$ & $M$ & No & Yes & Т8-T9 & No & Yes & Low & High \\
\hline II & 53 & $M$ & No & No & TIO-TII & No & Yes & Low & Low \\
\hline 12 & 51 & $M$ & No & No & LI-L2 & Yes & Yes & Low & Low \\
\hline 13 & 63 & $\mathrm{~F}$ & No & Yes & T9-TI0 & No & No & Low & Low \\
\hline 14 & 77 & $M$ & No & No & TII-TI2 & No & No & Low & Low \\
\hline 15 & 51 & $M$ & No & Yes & TII-TI2 & No & No & Low & High \\
\hline 16 & 34 & $M$ & No & No & TI2-LI & No & Yes & Low & Low \\
\hline 17 & 43 & $M$ & No & Yes & TIO-TII & Yes & Yes & Low & Low \\
\hline 18 & 55 & $M$ & No & No & TIO-TII & No & Yes & Low & Low \\
\hline 19 & 45 & $M$ & No & Yes & TIO-TII & Yes & No & Low & High \\
\hline 20 & 42 & $M$ & No & No & T9-TI0 & No & No & Low & High \\
\hline 21 & 49 & $M$ & No & Yes & TII-TI2 & Yes & Yes & Low & Low \\
\hline 22 & 52 & $M$ & No & Yes & TI2-LI & Yes & Yes & Low & Low \\
\hline 23 & $4 I$ & $\mathrm{~F}$ & No & No & LI-L2 & Yes & No & Low & Low \\
\hline 24 & 52 & $M$ & No & No & TII-TI2 & Yes & Yes & Low & Low \\
\hline 25 & 34 & $M$ & No & No & LI-L2 & Yes & Yes & Low & Low \\
\hline 26 & 67 & $M$ & No & No & L2-L3 & No & Yes & Low & Low \\
\hline 27 & 48 & $M$ & No & No & L3-L4 & Yes & No & Low & High \\
\hline 28 & 43 & $\mathrm{~F}$ & No & Yes & TII-TI2 & Yes & No & Low & High \\
\hline
\end{tabular}

Pts, Patients number;Trau-Histr, trauma history; Neuro-deficits, neurological deficits; Pseud-location, pseudarthrosis location 
Table 2 Clinical Details and Radiological Findings of Trans-vertebral Pseudarthrosis

\begin{tabular}{|c|c|c|c|c|c|c|c|c|c|}
\hline Pts & $\begin{array}{l}\text { Age } \\
(y r)\end{array}$ & Sex & $\begin{array}{l}\text { Trau- } \\
\text { Histr }\end{array}$ & $\begin{array}{l}\text { Neuro- } \\
\text { deficits }\end{array}$ & $\begin{array}{l}\text { Pseud- } \\
\text { location }\end{array}$ & $\begin{array}{l}\text { Extensive } \\
\text { destructive } \\
\text { lesion }\end{array}$ & $\begin{array}{l}\text { Vacuum } \\
\text { phenomenon }\end{array}$ & TI-image & T2-image \\
\hline I & 68 & $M$ & Yes & No & LI & No & Yes & Low & Low \\
\hline 2 & 52 & $M$ & Yes & No & $\mathrm{T} 12$ & No & No & Low & High \\
\hline 3 & 44 & $M$ & Yes & No & $\mathrm{T} 12$ & No & No & Low & Low \\
\hline 4 & 50 & $M$ & Yes & No & TIO & No & No & Low & High \\
\hline 5 & 52 & $M$ & Yes & No & TII & No & No & Low & Low \\
\hline 6 & 68 & $M$ & Yes & No & TII & No & Yes & Low & Low \\
\hline 7 & 53 & $M$ & Yes & No & LI & No & Yes & Low & High \\
\hline 8 & 45 & $M$ & Yes & No & TII & No & No & Low & Low \\
\hline
\end{tabular}

Pts, Patients number;Trau-Histr, trauma history; Neuro-deficits, neurological deficits; Pseud-location, pseudarthrosis location

\section{Histology}

Presence of the pseudarthrosis was confirmed at operation where a mobile segment was found in all 36 cases who received surgery. The operative findings of 12 patients with neurologic symptoms showed compression of the dural sac by the proliferated osteophytes and fibrotic tissues around pseudarthrosis, which had been ossified and adherent to neural tissues to variable degrees. The fibro-osseous tissue obtained from the sclerotic gap of posterior elements presented degenerated fibrocartilage with diffuse fibrosis. The pathology of extensive discovertebral lesions was consistent with a pseudarthrosis with fibrous tissue, small amounts of callus and sclerosis of the adjacent vertebral bone. There was only mild inflammatory cell infiltrate, which is thought to be secondary to mechanical damage.

\section{Discussion}

Andersson first described discovertebral destructive lesions (Andersson lession) of AS in $1937 . .^{9}$ Cawley et al..$^{10}$ classified these lesions into localized and extensive discovertebral destruction. It is now generally accepted that the extensive discovertebral destruction in AS is actually a pseudarthrosis. ${ }^{1-3,6,11,12}$ In our series, 18 of the 28 cases with trans-disc lesion showed characteristic of extensive discovertebral destructive lesion on plain film. The other 10 cases of trans-disc pseudarthrosis and all the 8 cases with trans-vertebral pseudarthrosis did not have this feature on plain film and their diagnosis needed further radiographic examination. So more than half of trans-disc thoracolumbar pseudarthrosis in AS have the feature of extensive discovertebral destruction and the cases with trans-vertebral thoracolumbar pseudarthrosis do not have this features.

The pseudarthrosis in AS is similar to a long tube bone nonunion, which occurs transversely through all three columns of the spine. Possible mechanisms for the formation of pseudarthrosis have been suggested. ${ }^{1}$ First, the segment of pseudarthrosis may escape fusion while other levels become ossified since the process of spinal ossification in AS is multifocal and discontinuous. Second, an acute fracture may occur through an already fused segment. Third, repeated stresses may invoke a fatigue fracture in the ankylosed spine as the thoracolumbar segments are subjected to the highest stress in a stiff kyphotic spine. As a result of a long arm ankylosed spine and persistent motion at the mobile site, the healing process stops and the bone fails to unite, which leads to pseudarthrosis. All the three mechanisms might be the causes of pseudarthrosis in our series. As shown in our series, all trans-vertebral pseudarthrosis have history of trauma, so acute fracture might been the cause of pseudarthrosis in this type.
Clinically, the presentations of pseudarthrosis include persistent back pain, neurological deficit and progressive deformity. The back pain may develop after a long quiescent period in the ankylosed spine. It is characterized by sharp and abrupt, which is intensified by exercise and relieved by rest. ${ }^{13}$ In the natural course of AS, the spinal symptoms tend to diminish with progressive ossification of the vertebrae. So the pseudarthrosis should be suspected when there is a recurrent back pain. Some cases might have a history of recent trauma as showed in all the 8 cases with trans-vertebral pseudarthrosis. The presence and degree of neurological deficit depends on the severity and location of pseudarthrosis. When the pseudarthrosis is above L1 where spinal cord locates, it is prone to cause neurological deficits. In our series, the 12 cases with neurological deficit were among the 28 cases with trans-disc type cases. The neurological deficits in these cases resulted from the proliferated osteophytes and fibrotic tissues around pseudarthrosis.

The pseudarthrosis in AS involves three columns of the spine and the plain radiographs alone could be not sufficient to show this condition due to distorted anatomy and osteopenia. ${ }^{14,15}$ Only 18 of our 36 cases had the feature of extensive discovertebral destructive lesion on radiograph. The other 18 cases, including 10 cases with transdisc type and all 8 cases with trans-vertebral type, needed further radiological examination for the diagnosis. It is also difficult in plain film to interpret changes in posterior element owing the heavily ossified spinal ligaments. CT, especially the multiplanar reformations, is superior to conventional X-ray examination in determining the extent of pseudarthrosis..$^{14}$ In the anterior column, CT with reconstruction can show irregular vertebral or discovertebral osteolysis with surrounding reactive sclerosis and the vacuum phenomena. In the middle column, the sclerotic lines were usually through the foraminae as showed in our series. In the posterior column, it can accurately demonstrate the sclerotic gap of the posterior elements or non-fusion of the facet joints. In our series, sequences of sagittal and coronal CT reconstruction showed the pseudarthrostic sclerotic gap from anterior column, across the foraminae, to the posterior elements. This continuous sclerotic gap is the characteristic changes in SP. MRI can supplement information on abnormal dural enhancement, epidural lesions and spinal canal stenosis. ${ }^{4-6}$ It is more useful in giving information about the state of the spinal cord and the nerve roots. ${ }^{16}$ Because it is a bony change in pseudarthrosis, CT is superior to MRI in describing this situation. The characteristic radiological appearances, especially the continuous sclerotic gap showed on the reformatted CT image, facilitate the diagnosis of SP in AS. 


\section{Conclusion}

Two types of thoracolumbar pseudarthrosis, the trans-disc type and trans-vertebral body type, can been classified in AS. More than half of the trans-disc types have the manifestation of extensive discovertebral destruction on plain film. When the trans-disc lesion locate above L1, it is prone to cause neurological deficit. Traumatic events usually precede in cases with the trans-vertebral type. Radiological examinations, such as CT with reconstruction and MRI, contribute to its diagnosis. The continuous sclerotic gap across all column of the spine showed on reformatted CT images is the characteristic of SP in AS. MRI is valuable in evaluating the spinal cord and soft-tissue injuries.

\section{Funding}

Shandong Province College Science and Technology Project (J15LL10, ZR2015050013) and Shandong Natural Research Foundation (ZR2016HM31) were received in support of this work.

\section{Acknowledgment}

None.

\section{References}

1. Fang D, Leong JC, Ho EK, et al. Spinal pseudarthrosis in ankylosing spondylitis. Clinicopathological correlation and the results of anterior spinal fusion. J Bone Joint Surg Br. 1988;70(3):443-447.

2. Kim KT, Lee SH, Suk KS, et al. Spinal pseudarthrosis in advanced ankylosing spondylitis with sagittal plane deformity: clinical characteristics and outcome analysis. Spine. 2007;32(15):1641-1647.

3. Park YS, Kim JH, Ryu JA, et al. The Anderson lesion in ankylosing spondylitis: distinguishing between the inflammatory and traumatic subtypes. J Bone Joint Surg Br. 2011;97(7):961-966.

4. Shih TT, Chen PQ, Li YW, et al. Spinal fractures and pseudoarthrosis complicating ankylosing spondylitis: MRI manifestation and clinical significance. J Comput Assist Tomogr. 2001;25(2):164-170.
5. Kurugoglu S, Mihmanli I, Kanberoglu K, et al. Destructive diskovertebral lesions in ankylosing spondylitis: appearance on magnetic resonance imaging. South Med J. 2001;94(4):837-841.

6. Eschelman DJ, Beers GJ, Naimark A, et al. Pseudoarthrosis in ankylosing spondylitis mimicking infectious diskitis: MR appearance. Am J Neuroradiol. 1991;12(6):1113-1114.

7. Dave BR, Ram H, Krishnan A. Andersson lesion: are we misdiagnosing it? A retrospective study of clinic-radiological features and outcome of short segment fixation. Eur Spine J. 2011;20(9):15031509 .

8. Langlois S, Cedoz JP, Lohse A, et al. Aseptic discitis in patients with ankylosing spondylitis: a retrospective study of 14 cases. Joint Bone Spine. 2005; 72(3):248-253.

9. Andersson O. Rontgenbilden vid spondyloarthritis ankylopoetica. Nord Med. 1937;14:2000-2002.

10. Cawley MID, Chalmers TM, Kellgren JH, et al. Destructive lesions of vertebral bodies in ankylosing spondylitis. Ann Rheum Dis. 1972;31(15):345-351.

11. Pettersson T, Lassonen L, Leirisalo-Repo M, et al. Spinal pseudoarthrosis complicating ankylosing spondylitis: a report of two patients. Br J Rheumatol. 1996;35(12):1319-1323.

12. Peh WC, Luk KD. Pseudoarthrosis in ankylosing spondylitis. Ann Rheum Dis. 1994;53(3):206-210.

13. Bron JL, de Vries MK, Snieders MN, et al. Discovertebral lesions of the spine in ankylosing spondylitis revisited. Clin Rheumatol. 2009;28(8):883-892.

14. Kenny JB, Hughes PL, Whitehouse GH. Discovertebral destruction in ankylosing spondylitis: the role of computed tomography and magnetic resonance imaging. Br J Radiol. 1990; 63(750):448-455.

15. Goldberg AL, Keaton NL, Rothfus WE, et al. Ankylosing spondylitis complicated by trauma: MR findings correrlated with plain radiographs and CT. Skeletal Radiol. 1993;22(5):333-336.

16. Toussirot E, Chataigner H, Pepin L, et al. Spinal cord compression complicating aseptic spondylodiscitis in ankylosing spondylitis. Clin Exp Rheumato. 2009;27(4):654-657. 